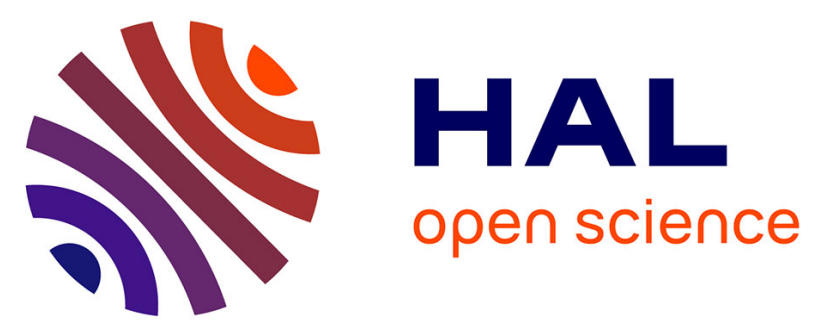

\title{
Macroscopic Quantum Tunneling of Magnetization of Single Ferrimagnetic Nanoparticles of Barium Ferrite
}

W. Wernsdorfer, E Bonet Orozco, Klaus Hasselbach, A. Benoît, D. Mailly, O Kubo, H Nakano, B. Barbara

\section{- To cite this version:}

W. Wernsdorfer, E Bonet Orozco, Klaus Hasselbach, A. Benoît, D. Mailly, et al.. Macroscopic Quantum Tunneling of Magnetization of Single Ferrimagnetic Nanoparticles of Barium Ferrite. Physical Review Letters, 1997, 79 (20), pp.4014. hal-01659970

\section{HAL Id: hal-01659970 https://hal.science/hal-01659970}

Submitted on 9 Dec 2017

HAL is a multi-disciplinary open access archive for the deposit and dissemination of scientific research documents, whether they are published or not. The documents may come from teaching and research institutions in France or abroad, or from public or private research centers.
L'archive ouverte pluridisciplinaire HAL, est destinée au dépôt et à la diffusion de documents scientifiques de niveau recherche, publiés ou non, émanant des établissements d'enseignement et de recherche français ou étrangers, des laboratoires publics ou privés. 


\title{
Macroscopic Quantum Tunneling of Magnetization of Single Ferrimagnetic Nanoparticles of Barium Ferrite
}

\author{
W. Wernsdorfer, ${ }^{1}$ E. Bonet Orozco, ${ }^{1}$ K. Hasselbach, ${ }^{2}$ A. Benoit, ${ }^{2}$ D. Mailly, ${ }^{3}$ O. Kubo, ${ }^{4}$ H. Nakano, ${ }^{5}$ and B. Barbara ${ }^{1}$ \\ ${ }^{1}$ LL Néel, CNRS, B.P. 166, 38042 Grenoble, France \\ ${ }^{2}$ CRTBT, CNRS, B.P. 166, 38042 Grenoble, France \\ ${ }^{3}$ LMM, CNRS, 196 Avenue H. Ravera, 92220 Bagneux, France \\ ${ }^{4}$ Toshiba Corporation, 8 Shinsugita-cho, Isogo-ku, Yokohama 235, Japan \\ ${ }^{5}$ Toshiba Glass Corporation, Yoshida-cho, Haibara-gun, Shizuoka 421, Japan
}

(Received 19 May 1997)

\begin{abstract}
Presented are switching field measurements of individual ferrimagnetic and insulating $\mathrm{BaFeCoTiO}$ nanoparticles containing about $10^{5} \mu_{B}$ at very low temperatures $(0.1-6 \mathrm{~K})$. For temperatures higher than $0.4 \mathrm{~K}$, the quantitative agreement with the Néel-Brown theory of thermal activated magnetization reversal allowed us to identify unambiguously the magnetization reversal of uniform rotation. Below $0.4 \mathrm{~K}$, strong deviations from this model are evidenced which are quantitatively in agreement with the predictions of the theory of macroscopic quantum tunneling of magnetization in the low dissipation regime. [S0031-9007(97)04527-4]
\end{abstract}

PACS numbers: 75.40.Gb, 75.50.Cc, 75.50.Tt

Observation of the quantum behavior of a macroscopic variable has remained a challenging problem [1]. Today, only very few macroscopic systems show agreement with theoretical predictions. Among them, there are Josephson junctions and SQUIDs where quantum tunneling between two macroscopically distinct current states has been observed [2]. After a slow evolution during the last decades, macroscopic quantum tunneling (MQT) in magnetism constitutes a very rich and fascinating field of research.

In single-domain nanoparticles, strong exchange interactions align the magnetic moments parallel to each other. These particles can be described by a macroscopic order parameter: the magnetic moment of the particle. Under the effect of an applied field, the rotation of such a moment can be described by an energy function having two minima which correspond to the two states of opposite moment direction. The energy barrier between these states is due to magnetocrystalline and/or shape anisotropy. At zero field, this energy barrier (proportional to the particle volume) is much too high to observe magnetization reversal. However, the barrier can be lowered by applying a magnetic field in the opposite direction of the particle magnetization. When the applied field is close to the switching field $H_{\mathrm{sw}}^{0}$, for which the barrier vanishes, thermal fluctuations can drive the system above the barrier. At very low temperature, it was shown theoretically that there is also the possibility of tunneling through the energy barrier [3].

Until now, quantum tunneling of magnetization (QTM) was mostly studied by measuring the temperature dependence of magnetic relaxation after an abrupt change in the applied magnetic field. At high temperature, when energy barriers are overcome by thermal activation, magnetic relaxation strongly depends on temperature. At low enough temperature, when thermal activation becomes extremely weak, magnetic relaxation should in principle vanish. Nevertheless, this is not always the case and sev- eral systems relax almost independently of temperature at low temperature [4]. This phenomenon was sometimes systematically attributed to QTM. However, as pointed out by Barbara et al. in Ref. [4], too naive interpretations of relaxation experiments in complex systems may lead to spurious conclusions, especially when the experiments have been done down to $2 \mathrm{~K}$ only. In particular, power-law distributions of energy barriers $n(E) \propto E^{-\delta}$ can simulate QTM [5]. Several processes giving such power-law distributions at low temperatures have been identified [6-9].

In order to avoid these complications, some groups tried to study the magnetization reversal of individual singledomain particles or wires. Most of these studies were done by using magnetic force microscopy at room temperature [10]. To our knowledge, only three groups could study magnetization reversal of small particles and wires at low temperatures [11-13]. However, their measurements lacked the ability to identify the classical regime described by the Néel-Brown theory of thermal activated magnetization reversal [14] and showed that such small particles and wires were still too complex (too large, defects due to surface roughness, oxidation, etc.). The agreement with the classical theory of Néel and Brown constitutes the precondition for the experimental observation of MQT.

In this Letter, we present single particle measurements on $10-20 \mathrm{~nm}$ particles of BaFeCoTiO. These particles are single-crystalline disks with hexagonal contour. We found that above $0.4 \mathrm{~K}$, the magnetization reversal of these particles is unambigously described by a thermally activated rotation of the particle's moment over a well defined anisotropy energy barrier, as originally proposed by Néel and Brown [14]. At lower temperature, strong deviations are evidenced which compare quantitatively to the predictions of the existing MQT theory without dissipation. Details on measuring technique, sample deposition, and a 
review of the Néel-Brown theory are reported in Ref. [15] and references therein.

The $\mathrm{BaFe}_{12-2 x} \mathrm{Co}_{x} \mathrm{Ti}_{x} \mathrm{O}_{19}$ nanoparticles have a strong uniaxial magnetocrystalline anisotropy depending on the Co-Ti substitution [16]. We studied compositions with $0 \leq x \leq 0.8$. The measurements presented in this Letter were obtained with $x=0.8$.

In order to confirm the single-domain character of our particles, we studied the magnetization reversal of each particle as a function of the applied field direction. Influence of temperature and time on the statistics of the magnetization reversal was studied by waiting time and switching field experiments. Via the waiting time measurements, we had direct access to the switching probability. At given temperature, the magnetic field was increased to a waiting field which was close to the switching field. Then, we measured the elapsed time until the magnetization switched. This process was repeated several hundred times, in order to obtain a waiting time histogram. The integral of this histogram gave the switching probability. Regarding switching field measurements, the applied field was ramped at a given rate $v=d H / d t$, and the field value was stored as soon as the sample magnetization switched. Then, the field ramp was reversed and the process was repeated. After several hundred cycles, switching field histograms were established, allowing us to obtain the mean switching fields $H_{\mathrm{sw}}$ and the width $\sigma$ (root mean square deviation). Both types of measurements can be studied as a function of the applied field direction which is defined by the angle $\theta$ between the easy axis of magnetization and the field direction.

In order to summarize the quasistatic magnetization measurements performed on a $10^{5} \mu_{B}$ BaFeCoTiO nanoparticle, called particle I, we present in Fig. 1 the angular dependence of the reversal field $H_{\mathrm{sw}}$. This dependence fits to the famous Stoner and Wohlfarth astroid showing that the magnetization reversal is a rotation at the unison [17]. The magnetocrystalline anisotropy field $\mu_{0} H_{a}$, found by these measurements for particles with a $\mathrm{Co}_{0.8} \mathrm{Ti}_{0.8}$ substitution, is about $0.4 \mathrm{~T}$.

Between 0.4 and $6 \mathrm{~K}$, waiting time and switching field measurements on all $\mathrm{BaFeCoTiO}$ nanoparticles were in complete agreement with the Néel-Brown theory: (i) exponential probabilities of not switching with mean waiting times following an Arrhenius law and (ii) mean switching fields and widths of the switching field distribution following the model of Kurkijärvi [18] [Eqs. (1) to (5) in Ref. [15]]. In Fig. 2, we demonstrate this agreement by a scaling plot of $H_{\mathrm{sw}}(v, T)$. For $T>0.4 \mathrm{~K}$, the data of $H_{\mathrm{sw}}(v, T)$ could be aligned on the master curve being a straight line. The adjustments allowed us to determine all parameters of the model of Kurkijärvi, namely, $E_{0}$ being the height of the energy barrier at $H=0, H_{\mathrm{sw}}^{0}$ being the switching field at $T=0 \mathrm{~K}$, and $\tau_{0}$ being the prefactor of the Arrhenius law [see Eq. (3) in Ref. [15]]. Knowing these parameters, the model of Kurkijärvi pre-

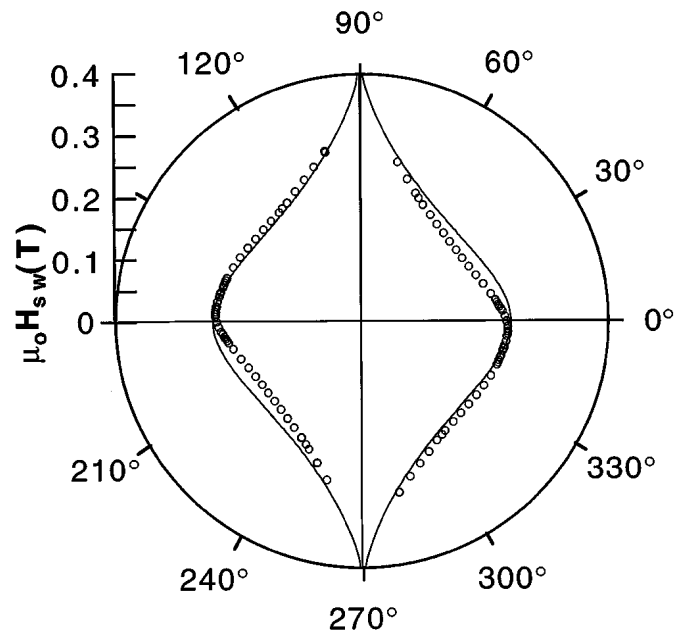

FIG. 1. Switching field $H_{\mathrm{sw}}\left(\theta^{\prime}\right)$ of the BaFeCoTiO particle I as a function of the angle $\theta^{\prime}$ measured between the applied field direction and the in-the-SQUID-plane-projected easy axis of magnetization, at $T=0.2 \mathrm{~K}$. The continuous line is the prediction of the Stoner-Wohlfarth model. Note that the easy axis of magnetization of this particle is out of the SQUID plane by about $20^{\circ}$ which leads to a rounding of the astroid near zero angle.

dicts the switching field distribution [the width $\sigma$ is given by Eq. (5) in Ref. [15]]. Again, a good agreement between theory and measurements was found for $T>0.4 \mathrm{~K}$ (Figs. 3 and 4).

This agreement could be confirmed by studying the angular dependence of $E_{0}$ following the prediction of the Stoner-Wohlfarth model $[17,19]$. The number of spins $S$ in the nanoparticle can be estimated by $S \approx$ $E_{0} /\left(2 \mu_{B} \mu_{0} H_{\mathrm{sw}}^{0}\right)$. We found $S \approx 10^{5}$ and $10^{6}$ for particles I and II, respectively. Finally, the angular dependence

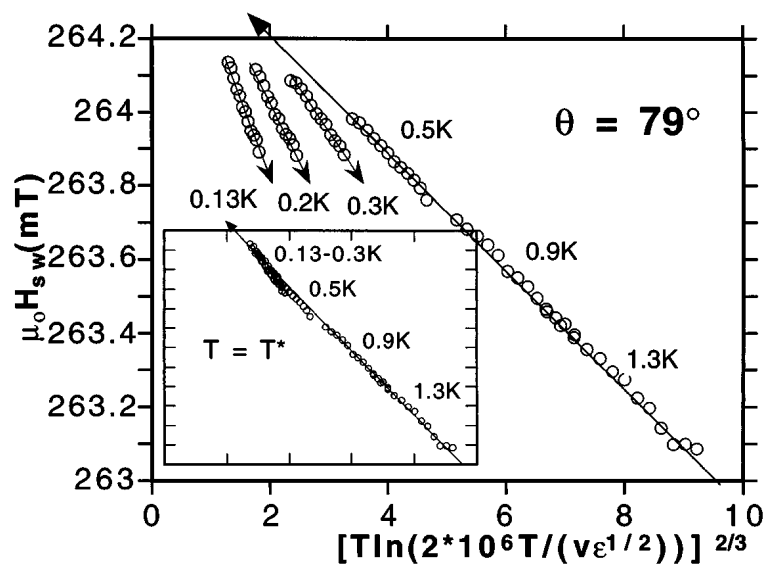

FIG. 2. Typical scaling plots of the mean switching fields $H_{\mathrm{sw}}$ for particle I. The applied field was swept at an angle of $\theta=79^{\circ}$. The measurements were done at field sweeping rates $v$ between $0.01-120 \mathrm{mT} / \mathrm{s}$ and temperatures between $0.13-$ $2.5 \mathrm{~K}$. Insets: Same data of $H_{\mathrm{sw}}(v, T)$ and same scales but the real temperature $T$ is replaced by an effective temperature $T^{*}$ which restores the scaling below $0.4 \mathrm{~K}$ (see Fig. 5). 


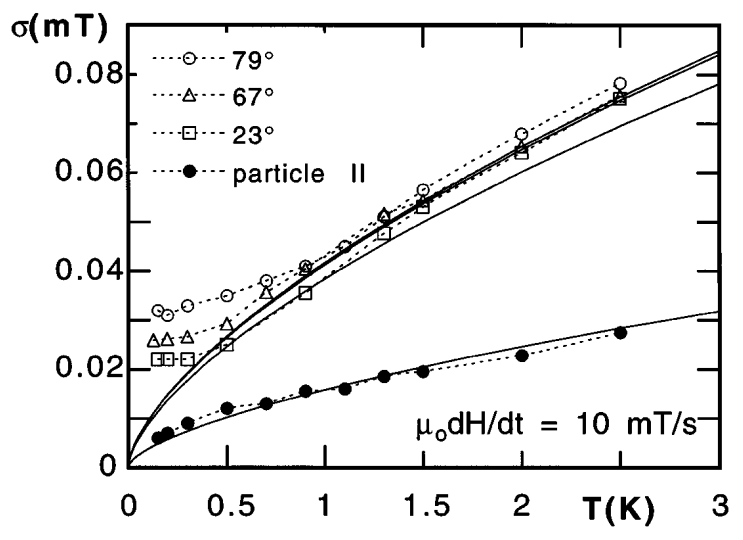

FIG. 3. Temperature dependence of the width of the switching field distribution $\sigma$ for $\mu_{0} d H / d t=10 \mathrm{mT} / \mathrm{s}$ and at three different angles of the applied field for particle I of about $10^{5} \mu_{B}$. Full points were measured on particle II of about $10^{6} \mu_{B}$ at $\theta \approx 20^{\circ}$. Lines: prediction of the Kurkijärvi model.

of $\tau_{0}$ followed well the prediction of Coffey et al. [20]. The phenomenological damping constant $\alpha$ from the Gilbert's equation could be found: $\alpha \approx 10^{-3}$ [21] which corresponds to the low damping regime, expected for insulating particles and justifying the comparison with the theory of QTM in the low dissipation regime.

Below $0.4 \mathrm{~K}$ several of the smallest particles showed strong deviations from the Néel-Brown model. These deviations were a saturation of the thermal dependence of $H_{\mathrm{sw}}$ and $\sigma$, and a faster field sweeping rate $(v=$ $d H / d t)$ dependence $H_{\mathrm{sw}}$ than given by the Néel-Brown model (see Figs. 2 and 3). In order to investigate the possibility that the low temperature deviations are due to an escape from the metastable potential by MQT, a common method is to replace the real temperature $T$ by an effective temperature $T^{*}(T)$ in order to restore the scaling plot. In the case of MQT, $T^{*}(T)$ should

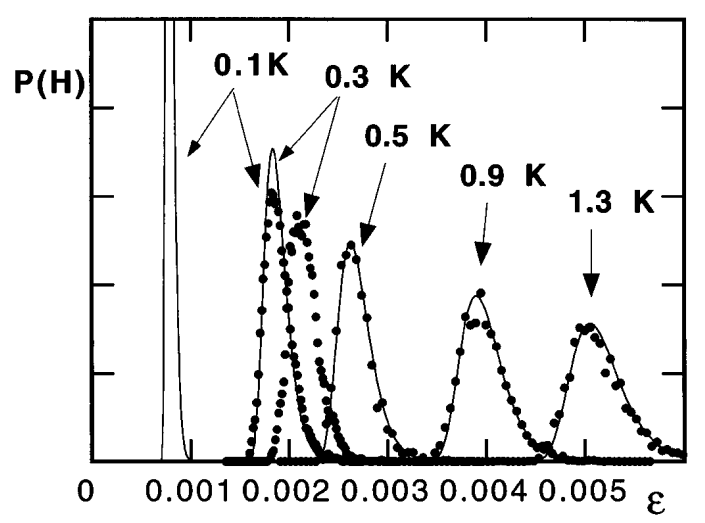

FIG. 4. Switching fields distributions for several temperatures as a function of $\varepsilon=1-H_{\mathrm{sw}} / H_{\mathrm{sw}}^{0}$. The applied field was swept at an angle of $\theta=79^{\circ}$ and at a rate of $\mu_{0} d H / d t=$ $60 \mathrm{mT} / \mathrm{s}$. The lines are predictions of the Kurkijärvi model showing a good agreement for $T>0.4 \mathrm{~K}$. All parameters of the Kurkijärvi model were found by the scaling plot in Fig. 2. saturate at low temperatures. Indeed, we found that the ansatz of $T^{*}(T)$ as shown in the inset of Fig. 5, can restore unequivocally the scaling plot demonstrated by a straight master curve (inset of Fig. 2). The flattening of $T^{*}$ corresponds to a saturation of the escape rate $\Gamma$ which is a necessary signature of MQT. As measurements at zero temperature are impossible, we investigated the effective temperature at our lowest measuring temperature and defined the crossover temperature between thermally activated and the quantum regime by $T_{c}=T^{*}$ at $T \approx$ $0.12 \mathrm{~K}$ (see inset of Fig. 5).

The angular dependence of the crossover temperature of a ferromagnetic particle [22] was considered by several authors [23-25]. As the BaFeCoTiO particles have a strong uniaxial anisotropy and weak in-plane anisotropy, we compared our measurements by taking into account only uniaxial anisotropy [24]:

$$
T_{c}(\theta) \propto \mu_{0} H_{a} \varepsilon^{1 / 4}|\cot \theta|^{1 / 6}\left(1+|\cot \theta|^{2 / 3}\right)^{-1},
$$

where $\mu_{0} H_{a}$ is the anisotropy field given in Tesla and $\varepsilon=$ $\left(1-H / H_{\mathrm{sw}}^{0}\right)$. The proportionally coefficient of Eq. (1) is of the order of unity and depends on the approach of the calculation [25]. Our measured angular dependence of $T_{c}(\theta)$ is in excellent agreement with this prediction (Fig. 5). The normalization value $T_{c}\left(45^{\circ}\right)=0.31 \mathrm{~K} \mathrm{com-}$ pares well with the theoretical value of about $0.2 \mathrm{~K}$. This quantitative agreement of the crossover temperature versus an external parameter strongly suggests MQT in these $\mathrm{BaFeCoTiO}$ particles.

Measurements on other particles with anisotropy between $H_{a}=0.3$ and $1 \mathrm{~T}$ showed that $T_{c}(\theta)$ is about proportional to $H_{a}$. Furthermore, we found that, for a given anisotropy field $H_{a}, T_{c}(\theta)$ decreases for bigger particles (see particle II in the inset of Fig. 5) which is also in agreement with Eq. (1) as $T_{c}(\theta)$ is proportional to $\varepsilon^{1 / 4}$

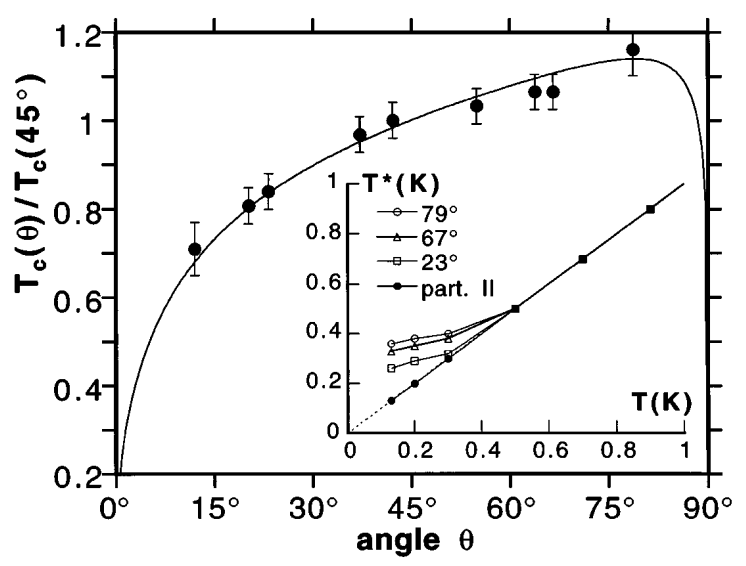

FIG. 5. Angular dependence of the crossover temperature $T_{c}$ for particle I. The line is given by Eq. (1). The values are normalized by $T_{c}\left(45^{\circ}\right)=0.31 \mathrm{~K}$. Inset: Effective temperature $T^{*}$ as a function of the real temperature $T$ at three different angles of the applied field. Full points were measured on the particle II, of about $10^{6} \mu_{B}$. 
(for a bigger particle, $\varepsilon$ must be smaller in order to measure a magnetization switching in the same time window, which is fixed by the field sweeping rate $v$ ). Finally, the width of the switching field distribution should be constant in the quantum regime which is also verified by our measurements (Figs. 3 and 4).

The agreement with the Néel-Brown theory was obtained recently for the first time on $20 \mathrm{~nm}$ Co particles of similar quality [15]. However, no clear quantum effects were found down to $0.2 \mathrm{~K}$. This was not surprising for two reasons: (i) The calculated crossover temperature for such a particle is smaller than $0.2 \mathrm{~K}$ and (ii) the dissipation effects due to conduction electrons may strongly reduce quantum effects which should not be the case in insulating $\mathrm{BaFeCoTiO}$ particles having a very small damping factor $\alpha=10^{-3}$.

The ultimate proof for MQT in a magnetic nanoparticle would be the observation of level quantization of its collective spin state which was recently evidenced in molecular $\mathrm{Mn}_{12}$ and $\mathrm{Fe}_{8}$ clusters having a collective spin state $S=10$ [26]. In our case of $S=10^{5}$, the field separation associated to the level quantization is rather small: $\Delta H=H_{a} / 2 S \approx 0.002 \mathrm{mT}$. Future measurements should focus on the level quantization of collective spin states of $S=10^{2}$ to $10^{4}$.

We acknowledge W. T. Coffey, E. Kennedy, H. Pascard, L. Sampaio, and A. Thiaville for very helpful discussions. This work is partially supported by DRET and Region Rhône-Alpes.

[1] A. J. Leggett, in Chance and Matter, Proceedings of the Less Houches Summer School, Session XLVI, edited by J. Souletie, J. Vannimenus, and R. Stora (North-Holland, Amsterdam, 1987), p. 395.

[2] R. F. Voss and R. A. Webb, Phys. Rev. Lett. 47, 265 (1981); J. Clarke, A. N. Cleland, M. H. Devoret, D. Esteve, and J. M. Martinis, Science 239, 992 (1988).

[3] J.L. van Hemmen and A. Sütö, Europhys. Lett. 1, 481 (1986); M. Enz and R. Schilling, J. Phys. C 19, 1765 (1986); 19, L711 (1986); E. M. Chudnovsky and L. Gunther, Phys. Rev. Lett. 60, 661 (1988).

[4] For a review, see B. Barbara et al., J. Magn. Magn. Mater. 140-144, 1825 (1995); J. Tejada and X. Zhang, J. Magn. Magn. Mater. 140-144, 1815 (1995).
[5] B. Barbara et al., in Proceedings of the International Workshop on Studies of Magnetic Properties of Fine Particles, Rome, 1991, edited by J.L. Dormann and D. Fiorani (North-Holland, Amsterdam, 1992), p. 235.

[6] B. Barbara and L. Gunther, J. Magn. Magn. Mater. 128, 35 (1993).

[7] B. Barbara et al., J. Magn. Magn. Mater. 136, 183 (1994).

[8] R. Ferré and B. Barbara, J. Magn. Magn. Mater. 140-144, 1861 (1995); R. Ribas and A. Labarta, J. Appl. Phys. 80, 5192 (1996).

[9] R. H. Kodama et al., Phys. Rev. Lett. 77, 394 (1996).

[10] For example, S. Gider et al., Appl. Phys. Lett. 69, 32693271 (1996).

[11] W. Wernsdorfer et al., J. Magn. Magn. Mater. 145, 33 (1995).

[12] K. Hong and N. Giordano, J. Magn. Magn. Mater. 151, 396 (1995).

[13] F. Coppinger et al., Phys. Rev. Lett. 75, 3513 (1995).

[14] L. Néel, Ann. Geophys. 5, 99 (1949); W. F. Brown, Phys. Rev. 130, 1677 (1963).

[15] W. Wernsdorfer et al., Phys. Rev. Lett. 78, 1791 (1997).

[16] O. Kubo, T. Ido, H. Yokoyama, and Y. Koike, J. Appl. Phys. 57, 15 (1985); O. Kubo, T. Ido, and H. Yokoyama, IEEE Trans. Magn. 23, 3140 (1987).

[17] E. C. Stoner and E. P. Wohlfarth, Philos. Trans. R. Soc. London, Ser. A 240, 599 (1948); L. Néel, C.R. Acad. Sci. 224, 1550 (1947).

[18] This model is a version of the Néel-Brown model for sweeping field measurements. J. Kurkijärvi, Phys. Rev. B 6, 832 (1972); L. Gunther and B. Barbara, Phys. Rev. B 49, 3926 (1994); A. Garg, Phys. Rev. B 51, 15592 (1995).

[19] Its variation is given by $E_{0} \propto|\cot \theta|^{1 / 3}\left(1+|\cot \theta|^{2 / 3}\right)^{-1}$, for $\varepsilon=\left(1-H / H_{\mathrm{sw}}^{0}\right) \ll 1$ and $0<\theta<90^{\circ}$.

[20] W. T. Coffey et al., Phys. Rev. B 52, 15951 (1995).

[21] We used Eq. (5.60) in L. J. Geoghegan, W. T. Coffey, and B. Mulligan, Adv. Chem. Phys. 100, 475 (1997).

[22] In fact, a BaFeCoToO particle is ferrimagnetic but the noncompensated magnetic moment is so large that it can be considered as a ferromagnetic one. A. Chiolero and D. Loss, Phys. Rev. B 56, 738 (1997).

[23] O. B. Zaslavskii, Phys. Rev. B 42, 992 (1990).

[24] M.-C. Miguel and E. M. Chudnovsky, Phys. Rev. B 54, 389 (1996).

[25] Gwang-Hee Kim and Dae Sung Hwang, Phys. Rev. B 55, 8918 (1997).

[26] J. Friedman et al., Phys. Rev. Lett. 76, 3820 (1996); L. Thomas et al., Nature (London) 383, 145 (1996); C. Sangregorio et al., Phys. Rev. Lett. 78, 4654 (1997). 